Paul Thissen (Bielefeld)

\title{
Tradition und Innovation in Schubarts Ideen zu einer Aesthetik der Tonkunst (1784/85)
}

Schubarts musikästhetisches Denken, das nicht nur in den Ideen zu einer Aesthetik der Tonkunst, sondern auch in den autobiografischen und publizistischen Schriften greifbar ist, wird in der Literatur zur Musik der zweiten Hälfte des 18. Jahrhunderts vor allem im Kontext des sog. „Sturm und Drang“ zwar immer wieder zitiert, ist aber, soweit ich es sehe, bisher nicht Gegenstand einer eigenständigen Untersuchung gewesen. Hierzu möchte der vorliegende Text einen ersten Beitrag leisten. Nach einigen einleitenden Bemerkungen zu Entstehung, Intention und Konzeption der Ideen, greife ich zentrale Aspekte der Schubart'schen Überlegungen auf, die sich in seinen Ausführungen zur Geschichte der Musik, zu Ausdruck und Stil, zum Verfall der Kirchenmusik und des Orgelspiels sowie zur Bach-Rezeption niederschlagen, und suche sie ästhetisch-historisch zu verorten. Leitendes Interesse ist es, Verbindungen zu tradiertem oder zeitgenössischem Gedankengut aufzuzeigen, vor allem aber originäre, in die Zukunft weisende Vorstellungen Schubarts hervortreten zu lassen, um so vielleicht eine Neubewertung seiner Bedeutung für die Geschichte musikästhetischen Denkens anstoßen zu können.

Bekanntermaßen wollte Alexander Gottlieb Baumgarten mit der Schrift Aesthetica ${ }^{1}$ die Theorie des Schönen und die Poetik als Lehre von der schöpferisch-künstlerischen Praxis um eine als eigenständige wissenschaftlich-philosophische Disziplin zu verstehende Theorie der sinnlichen Wahrnehmung und Erkenntnis ergänzen. Schubarts Schrift Ideen ist meines Wissens die erste Publikation, die den Terminus im Titel expressis verbis auf die Musik appliziert. So ist es nur naheliegend, dass Schubart gleich zu Beginn der Einleitung durchaus programmatisch anhebt:

„Man hat bisher behauptet, nur der mathematische Theil der Tonkunst lasse sich auf Grundsätze bringen; der aesthetische aber liege ganz und gar nicht im Gebiete der Kritik. Daher haben sich die Werke ersterer Art bis zum Ekel angehäuft, und von lezterer besitzen wir kaum einige matte, zitternde Versuche. [...] Nachstehende Abhandlung ist dazu bestimmt, diesen wichtigen Theil der Kunst zu bearbeiten, und die aesthetischen Grundsätze der Musik so deutlich als möglich darzustellen. "

Über die 1784/85 entstandenen Ideen, die Schubart während seiner vom württembergischen Herzog Carl Eugen ohne Anklage und Urteil veranlassten Gefangenschaft auf der sog. württembergischen Bastille, dem Hohenasperg, einem Sohn des ihm wohl gesonnenen Festungskommandanten Jacob von Scheeler diktierte, heißt es in Schubarts Charakter, dem von Schubarts Sohn Ludwig 1798 herausgegebenen dritten und letzten Teil von Schubart's Leben und Gesinnungen:

1 Frankfurt an der Oder 1750. Faks.-Repr. Hildesheim 1986

2 Christian Friedrich Daniel Schubart, Ideen zu einer Aesthetik der Tonkunst, in: C. F. D. Schubart, Gesammelte Schriften und Schicksale, 8 Bde., Stuttgart 1839. Faks.-Repr., 8 Bde. in 4 Bde., Hildesheim/New York 1972, Bd. 5, S. 11. Nachfolgend: Schubart, Ideen. 
„Er entwarf dieses Buch, wie er mir sagte, in der todten Einsamkeit seines ersten harten Gefängnisses, wollte das Resultat seines ganzen musikalischen Lebens, seiner Erfahrungen und seines eigenen Nachdenkens darin niederlegen, verschiedene ganz neue Ideen hier ausführen, und hatte sich selbst vorgenommen, das Werk gleich nach Beendigung seiner Lebensgeschichte noch einmal vorzunehmen, weiter und sorgfältiger auszuarbeiten, und bis auf unsere Zeiten fortzuführen. Er zweifelte nicht, daß es, so dargestellt, wie er die Idee im Herzen trug, etwas Eigenes und Ausgezeichnetes werden würde. "3

In diesem Zusammenhang verdient Erwähnung, dass Friedrich Schiller, der Carl Eugen in einem Brief an Körner als „absolutistischen Schwabenkönig“" bezeichnet, Schubart im Rahmen eines Besuchs einmal persönlich kennengelernt hat und sein Prosastück Spiel des Schicksals (1788) auf die Haft Schubarts zurückgreift. ${ }^{4}$

Die Rede von einem Entwurf des Buches muss insofern relativiert werden, als Schubart bereits in einem Brief vom 21. August 1770 an seinen Schwager Christian Gottfried Böckh,

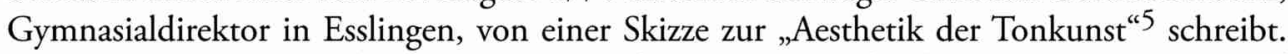
Das Vorhaben der Ausarbeitung und Weiterführung der Aesthetik bestätigt Schubarts Brief vom 24. Juni 1785 an die Ehefrau, zählt er hier doch die Aesthetik zu den Projekten, die er angesichts von Todesahnung ,in Ordnung zu bringen sucht ${ }^{\text {“ }}{ }^{6} \mathrm{Zu}$ einer Realisierung kam es allerdings nicht mehr, denn Schubart wurde am 11. Mai 1787 aus der Haft entlassen und durch Herzog Eugen als Theaterkritiker und Hofdichter in Stuttgart angestellt, wo er schließlich am 10. Oktober 1791 starb. Das über 400 Seiten umfassende Manuskript publizierte Schubarts Sohn Ludwig, von Vorabdrucken einiger weniger Passagen in verschiedenen Zeitschriften abgesehen, erst 1806, was zur Folge hatte, dass die unmittelbare Wirkung der Ideen gering blieb. Die bereits 1787 veröffentlichte Tonartencharakteristik, ${ }^{7}$ die noch in Ferdinands Hands Aesthetik der Tonkunst ${ }^{8}$ Spuren hinterlassen hat, ${ }^{9}$ ausgenommen, war aber auch die mittelbare posthume Rezeption eher schwach, wohl nicht zuletzt des vorläufigen Charakters der Schrift wegen - sie ist, wie der Titel schon vermuten lässt, tatsächlich eine eher aphoristische Sammlung von Ideen und Gedanken.

Dass das Urteil der Laien im 18. Jahrhundert immer mehr an Bedeutung gewann, dokumentiert u. a. der Untertitel vom Matthesons Schrift Das Neu-Eröffnete Orchestre (1713): „Universelle und gründliche Anleitung / Wie ein Galant-Homme einen vollkommenen Begriff von der Hoheit und Würde der edlen Music erlangen [...] möge." Während Mattheson aber immer noch auf eine gesellschaftlich privilegierte Schicht abzielt - immerhin ist das Ideal der Galanterie höfisch-aristokratischer Provenienz -, waren Schubarts literarische und journalistische Tätigkeiten stets von dem Bemühen geprägt, auch ein Publikum unterhalb der sog. Bildungsschicht zu erreichen. Hierin stand er dem „Volksdichter" Gottfried

3 Ludwig Schubart, Schubarts Karakter, in: Chr. F. D. Schubart, Gesammelte Schriften und Schicksale (wie Anm. 2), Bd. 2, S. 177.

4 Peter André Alt, Schiller. Leben - Werk - Zeit. Eine Biographie, Bd. 1, München 2000, S. 522 ff.

5 Chr. F. D. Schubart, Briefwechsel. Kommentierte Gesamtausgabe in drei Bänden, hrsg. von Bernd Breitenbruch, Konstanz 2006, Bd. 1, S. 190.

6 Ebd., Bd. 2, S. 173.

7 Chr. F. D. Schubart, Charakteristik der Toene, in: ders. (Hrsg.), Vaterländische Chronik, Stuttgart 1787. Faks.-Repr., hrsg. und mit einem Nachwort versehen von Hans Krauss, Heidelberg 1975, S. 55-66 und 63-64

8 Bd. 1, Leipzig 1837, Bd. 2, Jena 1841.

9 Siehe Matthias Tischer, Ferdinand Hands Aesthetik der Tonkunst. Ein Beitrag zur Inhaltsästhetik der ersten Hälfte des 19 Jahrhunderts, Sinzig 2004, S. 141 ff. 
August Bürger nahe,${ }^{10}$ mit dem er sich dem Göttinger Hainbund eng verbunden fühlte. Vor allem sein Musikunterricht war offenbar von Erfolg gekrönt, denn die Allgemeine Musikalische Zeitung urteilt 1799, die musikalische Kultur in Württemberg habe sich erstaunlich verbessert, „wozu der menschenfreundliche Schubart, zu welchem aus der Nähe und Ferne bei 100 wallfahrteten und denen er Lehrer und Kapellmeister war, die erste Impulsion gab". ${ }^{11}$ Nach der Entlassung aus der Haft setzt Schubart nicht nur mit Hilfe der von ihm bereits in den Jahren 1774 bis 1777 herausgegebenen Deutschen Chronik, einem zweimal wöchentlich erscheinenden Periodikum, in dem, wie es im Vorwort heißt, „Politik, Literatur, Dichtkunst, Musik und bildende Künste miteinander abwechseln ", 12 Popularisierung und Bildung fort, sondern befördert sie zudem durch öffentliche Lesungen und Vorträge zur Ästhetik, ${ }^{13}$ was umso mehr hervorzuheben ist, als die Dichotomie zwischen Gebildeten und Ungebildeten noch eine Grundstruktur der Gesellschaft des 19. Jahrhunderts blieb. Auch Schubarts Ideen verdanken ihre Entstehung seinem Credo, man müsse „Weisheit und Geschmack als allgemeine Ware für jedermanns Kauf anbieten “. ${ }^{14}$ Geschmack ist die Fähigkeit, die Empfindung des Wohlgefallens wahrzunehmen, in der ästhetischen Theorie der Aufklärung „das Vermögen, das Schöne zu empfinden, so wie die Vernunft das Vermögen ist, das Wahre, Vollkommene und Richtige zu erkennen". ${ }^{15}$ Baumgarten spricht von der „scientia cognitionis sensitivae“. ${ }^{16}$ Ziel der Ästhetik sei, so Baumgarten, die Vervollkommnung der sinnlichen Erkenntnis des Menschen, die Schulung des Geschmackssinns, und genau dies ist auch Schubarts Intention.

Die Ideen bestehen aus zwei großen Hauptteilen; dies sind die „Skizzierte Geschichte der Musik“ sowie „Die Grundsätze der Tonkunst" mit lexikalischen Artikeln zu den verschiedenen Instrumenten - nebst einem interpolierten Text „Ideal eines Orgelspielers“ - , zu den „musikalischen Kunstwörtern“ - gleichermaßen wie die „Geschichte" offenbar angeregt durch entsprechende Teile in der Violinschule Leopold Mozarts - und dem „musikalischen Kolorit" sowie eine kurze Stillehre und einige Gedanken zum "musikalischen Ausdruck“, die mit der berühmten Tonartencharakteristik schließen.

\section{Geschichtsbild}

In gleicher Weise wie Mattheson, der die Musik in der Hierarchie der Künste auf der Basis des Postulats ihres nichtmimetischen Ursprungs privilegiert - der Ursprung der Musik sei "primo loco in Gott selbst / secundo in der eigentlichen Natur" 17 zu finden, so argumentiert Mattheson -, geht Schubart zu Beginn des Abschnitts zur Geschichte, dessen relativ breite Entfaltung ein Reflex auf Herders Vorstellung sein mag, das ästhetische Subjekt bedürfe ei-

10 Siehe Bernd Jürgen Warneken, Schubart. Der unbürgerliche Bürger, Frankfurt a. M. 2009, S. 15. Nachfolgend: Warneken, Schubart.

11 Zitiert nach: ebd., S. 287f.

12 Deutsche Chronik auf das Jahr 1774, hrsg. von Chr. F. D. Schubart, Augsburg 1774. Faks.-Repr. Heidelberg 1974, S. III.

13 Warneken, Schubart, S. 161.

14 Schubart, Ideen, S. 114.

15 Johann Georg Sulzer, Allgemeine Theorie der schönen Künste. Im einzeln, nach alphabetischer Ordnung der Kunstwörter auf einander folgenden, Artikeln abgehandelt. Theil I, Leipzig 1771, S. 461.

16 Aestehtica [wie Anm. 1], S. 1.

17 Johann Mattheson, Das Neu-Eröffnete Orchestre [1713]. Faks.-Repr., hrsg. von Dietrich Bartel, Laaber 2002, S. 306. 
nes ausgeprägten Geschichtsbewusstseins, ${ }^{18}$ mit der Nachahmungsästhetik hart ins Gericht. $\mathrm{Da}$ auch noch um die Jahrhundertmitte die Bemühungen, auf der Basis der Mimesistheorie den ästhetischen Rang der Musik zu fundieren, ergebnislos blieben, muss betont werden, dass Schubart hier keinesfalls, wie es zunächst scheinen könnte, einen verspäteten Diskurs führt.

„Es ist also kindisch und ganz und gar gegen die Würde der Menschheit, wenn man [...] annehmen wollte, der Mensch hätte das Singen von den Vögeln gelernt oder Musik sei nachahmende Kunst. ${ }^{\text {"19 }}$

Mit diesen Worten bezieht Schubart explizit Stellung nicht nur gegen Batteux ${ }^{20}$ und d'Alembert, ${ }^{21}$ der die Musik im System der Künste zudem noch auf dem letzten Rang sieht, ${ }^{22}$ sondern zudem ganz offensichtlich gegen Leopold Mozart, der seinen „Versuch einer kurzen Geschichte der Musik“, dem II. Kapitel der Violinschule, die Entstehung der Musik mit der Nachahmung von Naturlauten in Verbindung bringt: „Adam“, so Mozart, „hörte den Gesang verschiedener Vögel; er vernahm eine abwechselnde Höhe und Tiefe durch das Gepfeife des zwischen die Bäume dringenden Windes. " 23 Dass Schubart in der Ablehnung der Nachahmungstheorie nicht so sehr, wie es zunächst den Anschein zu haben scheint, von Mattheson, als vielmehr von Klopstock beeinflusst war, ist durchaus denkbar, war doch in Schubarts Leben kaum ein zweites Ereignis so prägend wie die Begegnung des 12-Jährigen mit dem Messias, dessen Verfasser Schubart als Idealtypus eines Originalgenies gilt, das „nicht nur“, so Schubart, „den größten Genies, die jemals gelebt haben, einem Homer, Shakespeare, Dante und Milton vollkommen“ gleichkomme, sondern „sie an Empfindung und Erhabenheit" 24 übertreffe.

Als musikhistorische Werke, auf die er bereits zurückgreifen konnte, erwähnt Schubart die in drei Bänden erschienene Geschichte der Musik Padre Martinis (Storia della musica, 1759-1781) sowie die 1776 in fünf Bänden erschienene Musikgeschichte ( $A$ general history of science and practice of music) von John Hawkins. Dass Schubart diese Publikation trotz ihrer häufig angesprochenen „mangelhaften Disposition“ 25 als „das wichtigste Werk, was über diesen Gegenstand jemals geschrieben wurde" ${ }^{26}$ wertet, $A$ general History of Music (1776-1789) Charles Burneys dagegen, den er 1772 in Ludwigsburg persönlich kennen gelernt hatte, des nicht originalen Urteils und nicht viel sagenden ästhetischen Gefühls zeiht, ${ }^{27}$ scheint weniger sachlich begründet als vielmehr durch die Tatsache motiviert zu sein, dass

18 Die Urteilskraft, so schreibt Herder in Kritische Wäldchen, oder Betrachtungen über die Wissenschaft und Kunst des Schönen. Viertes Wäldchen, solle „unendlich“ werden „wie die Geschichte der Menschheit“. Johann Gottfried Herder, Sämtliche Werke, hrsg. von B. Suphan, Berlin 1877ff., Bd. 4, S. 41.

19 Schubart, Ideen, S. 13.

20 Charles Batteux, Traité des Beaux arts, reduits à un même princip, Paris 1746.

21 Jean le Rond d'Alembert, Discours préliminaire, in: Encylopédie ou Dictionnaire raisonné des sciences, des arts et des métiers, hrsg. von Denis Diderot und J. le Rond d'Alembert, Paris 1751, I-XLV.

22 "Enfin la Musique, qui parle à la fois à l'imagination et aux sens, tient le dernier rang dans l'ordre de l'imitations". Ebd., S. 36.

23 Leopold Mozart, Versuch einer gründlichen Violinschule, Augsburg 1756. Faks.-Repr., hrsg. von Greta Moens-Haenen, Kassel u. a. 1995, S. 13.

24 Chr. F. D. Schubart, Klopstock, in: Vermischte Schriften. Erster Theil, hrsg. von L. Schubart, in: Chr. F. D. Schubart., Gesammelte Schriften [wie Anm. 2], Bd. 6, S. 36

25 Bernhard Schrammek, Hawkins, Sir John, in: MGG2, Personenteil, Bd. 8, Kassel usw. 2002, Sp. 897.

26 Schubart, Ideen, S. 201.

27 Ebd., S. 265. 
Burney, wie Schubart beklagt, den Deutschen „bloß Kunstfertigkeit und Fleiß“ attestiert, „das musikalische Genie“ ${ }^{28}$ aber abspricht.

Die Musikgeschichten Martinis und Burneys antizipieren zumindest ansatzweise Hegels Konzept von Universalgeschichte und lassen sich mit Maßstäben der modernen Historiografie messen, wohingegen Schubarts Musikgeschichte, wie die Überschrift andeutet, allenfalls eine grobe Skizze darstellt, die allerdings, und damit weist Schubart über das verbreitete musikhistorische Denken seiner Zeit deutlich hinaus, nicht die zeitgenössische Musik zum Maßstab auch für die Musik vergangener Zeiten erhebt und sich einer Abwertung der alten Musik enthält. Dass Schubarts Sicht von Herders Vorstellung, auch vergangene Kulturen seien nach den ihnen angemessenen Kriterien zu bewerten, beeinflusst war, steht außer Frage, war Schubart doch ein begeisterter Leser der Schriften Herders. Während Forkel z. B. ein Geschichtsbild entwarf, das die Musik in einem beständigen Fortschritt sieht und den Parameter Harmonik als Beweis der Überlegenheit der zeitgenössischen Musik über die Musik vergangener Zeiten heranzieht, wird bei Schubart ein historiografisches Prinzip greifbar, das man bisher erst bei August Wilhelm Ambros wirksam zu werden glaubte, ein Historismus nämlich, der heuristisch davon ausgeht, dass die Kulturen der Vergangenheit nicht als Vorstufen späterer Kulturen, sondern vielmehr als Gebilde eigenen Rechts anzusehen sind. So nennt er den David des Alten Testaments „eine[n] der größten Musiker", 29 und über die griechische Musik urteilt er, „Einfalt und Erhabenheit“ - für Schubart zentrale Kriterien für den ästhetischen Rang von Musik - seien „ganz gewiss der Hauptcharakter der griechischen Musik gewesen“. ${ }^{30}$ „[R]egelrecht bahnbrechend“31 ist aufgrund seiner Akzeptanz der „Einstimmigkeit der griechischen Musik “32 mithin bereits das historische Denken Schubarts und nicht erst, wie Frank Hentschel in seiner äußerst lesenswerten Studie zur Musikhistoriografie meint, das August Wilhelm Ambros. ${ }^{33}$

1754 formuliert Marpurg den Wunsch nach einer „Historie der Tonkunst“, in der vor allem „der einer jeden Nation besonders eigene Geschmack und die Verbesserung desselben [...] in gehöriger Verbindung "34 aller nur denkbaren Aspekte geschehen müsse. Diesem Anspruch sucht Schubart vor allem im Hinblick auf die bisher entweder vernachlässigte oder in seinen Augen nicht angemessen beurteilte deutsche Musik nachzukommen. Deren - den größten Raum einnehmende und durchaus apologetisch zu verstehende - Darstellung „nach Art der Malerschulen " 35 ist offenbar den politischen Gegebenheiten verpflichtet - Deutschland war nach dem Westfälischen Frieden bekanntlich in ca. 300 Territorien geteilt - und geht auf die musikalische Tradition der verschiedenen Höfe ein. Auf die Bedeutung des hierbei von Schubart in den Blick genommenen Nationalstils komme ich unten zu sprechen.

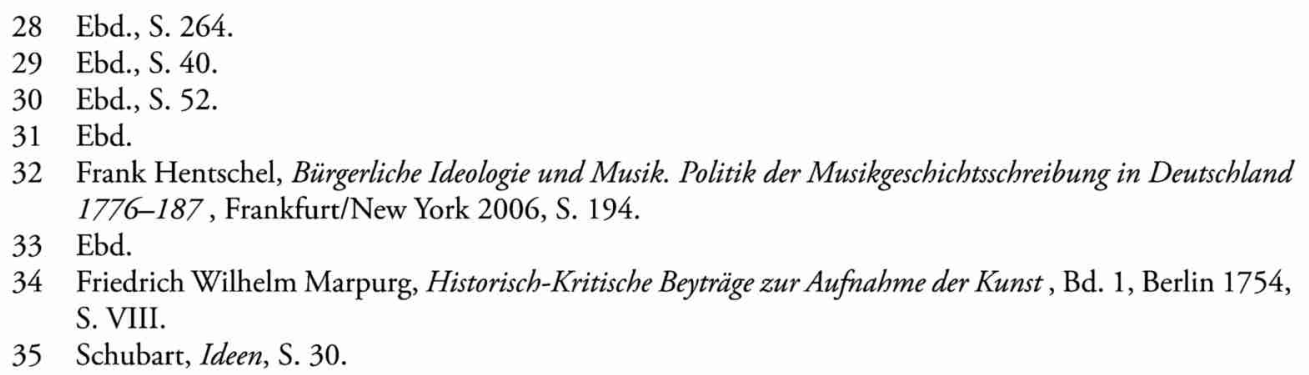

34 Friedrich Wilhelm Marpurg, Historisch-Kritische Beyträge zur Aufnahme der Kunst, Bd. 1, Berlin 1754, S. VIII.

35 Schubart, Ideen, S. 30 


\section{Ausdruck}

„Er liebt, er zürnt, er klagt, er tobt, raset, bebet, verflucht, er lacht, er weint, er mischt sich ins Halleluja der Engel und ins dumpfe Getöse der Harfen des Todes vom Donner gespalten [...].“36

Diese Worte, mit denen Schubart innerhalb der „Skizzierte[n] Geschichte der Musik“, gegen die Nachahmungsästhetik argumentierend, darlegt, was der Mensch mit Hilfe von „sieben Tönen“ hervorzubringen in der Lage sei, mögen das Verständnis Eggebrechts der Person Schubarts als „das Urbild des Stürmers und Drängers“, als „Expressionstypus par excellence " 37 befördert haben. Tatsächlich hebt Schubart zu Beginn des letzten Abschnitts der Ideen, „Vom musikalischen Ausdruck“ überschrieben, in besonderer Weise hervor, der „musikalische Ausdruck“ sei „gleichsam die goldene Achse, um welche sich die Aesthetik der Tonkunst dreht" und nennt als dessen zentralen Eigenschaften "Richtigkeit, Deutlichkeit und Schönheit". ${ }^{38}$ Und der V. Abschnitt der ebenfalls auf dem Hohenasperg verfassten Klavierrecepte hebt an mit der These:

„Ohne Ausdruck ist alle Musik Gefasel, und dein Gespiele ist nicht Herzenssprache, sondern unverständliches Gewäsche. “39

Von einem Organisten, der in einer angesehenen Stadt mit einer guten Orgel den Volksgesang zu begleiten hat, fordert Schubart ein „[a]ndachterweckendes Arioso unter dem heiligen Abendmahle, [...] klagende, durch alle Herznerven wühlende Phantasie an Bußtägen, und lautes Aufjauchzen mit allen Registern an hohen Festtägen [...]“. ${ }^{40}$ Und er fährt fort mit einem Ambrosius-Zitat, das, die Voraussetzung für eine Realisierung dieser Forderungen nennend, C. P. E. Bachs berühmtes Diktum ,indem ein Musickus nicht anders rühren kann, er sey dann selbst gerührt “ ${ }^{41}$ zu antizipieren scheint, aber auf die Sentenz „Si vis me flere, dolendum est primum ipsi tibi“ aus Horaz' Ars poetica zurückgeht ${ }^{42}$ und, so Schubart, „auch beim Orgelspiel wahr" sei: „Willst du der Gemeinde im Gesange vorstehen [...], so mußt du erst selbst fühlen, was du singst". ${ }^{43}$ Möglicherweise wurde Schubart zur Aufnahme dieser Sentenz angeregt durch einen Abschnitt aus dem Dialog Von der Darstellung seines Idols Klopstock, wo es heißt, der Dichter müsse selbst „herzlichen Anteil“ nehmen an dem, was er sagt, um den Zuhörer „zu gleicher Teilnehmung“ “44 reizen zu können. Ziel ist es also, um mit C. P. E. Bach zu sprechen, sich „selbst in alle Affecten setzen zu können“, welche man beim Zuhörer bzw. Kirchenbesucher erregen will. D. h. der Interpret schlüpft gleich einem

36 Ebd., S. 36.

37 Hans Heinrich Eggebrecht, Das Ausdrucks-Prinzip im musikalischen Sturm und Drang, in: Musikalisches Denken. Aufsätze zur Theorie und Ästhetik der Musik, Wilhelmshaven 1977, S. 91.

38 Schubart, Ideen, S. 281.

39 In: Chr. F. D. Schubart, Vermischte Schriften. Erster Theil [wie Anm. 24], S. 72.

40 Chr. F. D. Schubart, Schubart's Leben und Erinnerungen, in: ders., Gesammelte Schriften [wie Anm. 2], Bd. 1, S. 98 f. Nachfolgend: Schubart, Schubart's Leben

41 Versuch über die wahre Art das Clavier zu spielen, Teil 1, Berlin 1753, Teil 2, Berlin 1762. Faks.-Repr. mit den Ergänzungen der Auflagen Leipzig 1787 [Teil 1] und 1797 [Teil 2], hrsg. von Wolfgang Horn, Kassel usw. 1994, S. 122.

42 Siehe Carl Dahlhaus, „Si vis me flere ... “ [1972], in: ders., Klassische und romantische Musikästhetik, Laaber 1988, S. 28.

43 Schubart, Schubart's Leben, S. 99.

44 In: Friedrich Gottlob Klopstock, Ausgewählte Werke, hrsg. von Karl August Schleiden, 2 Bde., München 1962, Bd. 2, S. 1035. 
Schauspieler in eine Rolle, womit ein solchermaßen verstandener Ausdruck immer noch unverkennbar in der Tradition der überkommenen Affektenlehre steht.

Wenn Schubarts Sohn aber das Fantasieren seines Vaters auf der Orgel beschreibt, scheint eine neue Stufe erreicht zu sein:

„Er fing hier gewöhnlich mit vieler Ruhe an; er erwärmte sich aber [...] und gerieth sodann in ein Feuer, das Stundenlang anhalten konnte, und worin er sich, und alles, was um ihn vorging, völlig vergaß. Er sah nichts, hörte nichts, achtet auf nichts, - war ganz in seinem Thema verloren und untergegangen. “45

Die Ähnlichkeit mit Beschreibungen des fantasierenden C. P. E. Bach sind unübersehbar. ${ }^{46}$ Schubart scheint hier aus der Realität herauszutreten und, um seine prägnante Formulierung aus den bereits erwähnten Klavierrecepten zu gebrauchen, mit dem Instrumentalspiel seine „Ichheit auch in der Musik herauszutreiben". ${ }^{47}$ Mit einer Deutlichkeit, die nichts zu wünschen übrig lässt, hebt dieses Schubart'sche Diktum eine qualitativ neue Ebene ins Wort, geht es nun doch tatsächlich um den ganz persönlichen Ausdruck eines biografisch fassbaren Subjekts, womit das obige Zitat [„Ohne Ausdruck ist alle Musik Gefasel“] ein ganz neues Gewicht bekommt, denn es zeigt, dass die Grenze zur zu Beginn des 20. Jahrhunderts so viel gescholtenen Subjektivität in der Musik des 19. Jahrhunderts schon in den 80er Jahren des 18. Jahrhunderts überschritten wurde. In Schubart nun aber deshalb einen Vorboten der Romantik zu sehen, ${ }^{48}$ dürfte eine Übertreibung sein. Er ist zwar Zeuge des Willens eines individuellen Ichs, sich in der Musik auszudrücken, keinesfalls aber Repräsentant einer gegen Ende des 18. Jahrhunderts zu Ehren gelangenden Ästhetik des Unbestimmten. Schubarts Überzeugung, „der musikalische Ausdruck“ sei „so bestimmt, daß [...] er es doch an $\mathrm{Ge}$ nauigkeit dem poetischen und pittoresken Ausdrucke weit zuvorthut ${ }^{\text {“ } 49}$ und seine hieraus resultierende beharrliche Forderung nach Deutlichkeit des Ausdrucks ${ }^{50}$ lassen ersichtlich werden, dass er auf anschauliche Weise Gefühlsinhalte vermitteln wollte. Die Behauptung Hammersteins, mit Schubart kündige sich eine „poetisch-romantische Musikbeschreibung an ", 51 wird dessen ästhetischem Denken kaum gerecht, ist die romantische Musikästhetik doch ganz wesentlich konstituiert durch den von Carl Philipp Moritz grundgelegten Autonomiegedanken ${ }^{52}$ und der davon abhängigen Metaphysik der Instrumentalmusik. Der Vorrangstellung einer autonomen, rein instrumentalen Musik und ihrer kontemplativen, von einer religiösen Haltung geprägten Wahrnehmung wird man in Schubarts Schriften jedoch an keiner Stelle begegnen. Nichts ist der romantischen Musikästhetik mehr fremd als die für Schubart so zentrale Darstellung von individuellen, konkret benennbaren Gefühlszuständen, sind doch, wie Ludwig Tieck 1799 in den Phantasien über die Kunst schreibt,

45 Schubarts Karakter [wie Anm. 3], S. 158.

46 Vgl. Noch ein Bruchstück aus J. F. Reichardt's Autobiographie. Sein erster Aufenthalt in Hamburg, in: AmZ 16, Nr. 2 (12.1.1814), Sp. 28.

47 Chr. F. D. Schubart, Klavierrecepte [wie Anm. 39], S. 73.

48 Vgl. Kurt Honolka, Schubart: Dichter und Musiker, Journalist und Rebell. Sein Leben, sein Werk, Stuttgart 1985, S. 80.

49 Schubart, Ideen, S. 385.

50 Ebd., S. 377.

51 Reinhold Hammerstein, Christian Friedrich Daniel Schubart, ein schwäbisch-alemannischer DichterMusiker der Goethezeit, Diss. Freiburg i. Br. 1940, S. 124.

52 Vgl. Carl Philipp Moritz, Versuch einer Vereinigung aller schönen Künste und Wissenschaften unter dem Begriff des in sich selbst Vollendeten (1785), in: ders., Schriften zur Ästhetik und Poetik, hrsg. von Hans Joachim Schrimpf, Tübingen 1962, S. 3. 
die „Töne der Instrumente [...] eine Welt für sich selbst“ ${ }^{53}$ Diesen entscheidenden Aspekt prägt Schubart, im Gegensatz zur englischen Ästhetik der Mitte des 18. Jahrhunderts (Charles Avison), noch nicht einmal im Ansatz aus, was nicht zuletzt auch in seiner musikalischer Sozialisation gründen mag, die sehr stark durch die Oper geprägt war. An den Besuch der Oper Fetonte Jommellis, der einem musikalischen Erweckungserlebnis gleichkommt, erinnert er sich später mit den Worten:

„Man stelle sich einen so feuerfangenden Menschen vor, als ich war, dessen Haupthang die schönen Künste, sonderlich die Tonkunst, gewesen, und der noch nie ein treffliches Orchester gehört, noch nie eine Oper gesehen hatte, diesen Menschen stelle man sich vor - wie er schwimmt in tausendfachen

Wonnen, indem er hier den Triumph der Dichtkunst, Malerei, Tonkunst und Mimik vor sich sah. "54

Dem Erzielen der von Schubart immer wieder beschworenen Deutlichkeit des Ausdrucks dient seine die Ideen beschließende Tonartencharakteristik, die in Wien, wie Andreas Krause wahrscheinlich machen konnte, von Komponisten wie Schubert positiv rezipiert und angewandt wurde. ${ }^{55}$ Diese Wertschätzung mutet erstaunlich an. Nimmt man die ungefähr zeitgleich mit Schubart entstandenen Ausführungen Wilhelm Heinses zum Charakter von Tonarten in den Blick, so wird deutlich, dass Heinse sich „um ein theoretisches Modell für den Beweis der Existenz von Tonartencharakteren " 56 bemüht, während Schubart sie unbegründet apodiktisch in den Raum stellt, was um so schwerer wiegt, als Heinse die ungleichstufige Temperierung als Voraussetzung für die unterschiedlichen Ausdruckscharaktere der Tonarten sieht, wohingegen Schubart zumindest im Hinblick auf die Orgel für eine gleichstufige Temperierung plädiert. Zweifellos bedeutet ein solcher Befund - wiewohl der Verfasser als Liederkomponist sich an ihr zu orientieren scheint - eine Relativierung der Bedeutung der Schubart'schen Tonartencharakteristik. So liegt ihr Wert wohl, wie der Schubart-Biograf Bernd Jürgen Warneken hervorhebt, „vor allem darin, dass er [sic] einen Einblick in die zeitgenössische ,Kultur der Stimmungen' erlaubt [...], in die Lebensthemen, mit denen Gefühlsregungen vor allem verbunden waren, in die Konventionen der Gefühlsbenennung und der Zuordnung bestimmter Gefühle zu bestimmten Gruppen (,fromme Weiblichkeit'). ${ }^{\text {"57 }}$ Genau darauf zielte offenbar bereits Schumann ab, der in Schubarts „Charakteristik der Töne“ auch „viel Zartes und Poetisches" 58 fand.

\section{Stil}

Schubarts Anmerkungen zum Stil stehen in einer über Johann Mattheson bis auf Mario Scacchi zurückreichenden Tradition, stellen aber alles andere als eine konsistente zeitgenös-

53 Ludwig Tieck/Wilhelm Heinrich Wackenroder, Phantasien über die Kunst, für Freunde der Kunst, hrsg. von Ludwig Tieck, Hamburg 1799, S. 241.

54 Schubart, Schubart's Leben, S. 83.

55 Andreas Krause, Die Klaviersonaten Franz Schuberts, Kassel u. a. 1992, S. $99 f f$. Grundlegend für das Thema „Tonartencharakter": Wolfgang Auhagen, Studien zur Tonartencharakteristik in theoretischen Schriften und Kompositionen vom späten 17. bis zum Beginn des 20. Jahrhunderts, Frankfurt a. M. 1983.

56 Kerstin Jaunich, Tonsysteme und Tonartencharakteristik in Wilhelm Heinses musikalischen Schriften, in: „Seelenaccente“- „Ohrenphysiognomik“. Zur Musikanschauung E. T. A. Hoffmanss, Heinses und Wackenroders, hrsg. von Werner Keil und Charis Goer (= Diskordanzen. Studien zur neueren Musikgeschichte, hrsg. von Werner Keil, 8), Hildesheim/Zürich/New York 2000, S. $274 \mathrm{f}$.

57 Warneken, Schubart, S. 7 ,

58 Robert Schumann, Charakteristik der Tonarten [1835], in: ders., Gesammelte Schriften über Musik und Musiker, hrsg. von Martin Kreisig, Leipzig 1854, Bd. 1, S. 180f. 
sische Stillehre dar, wie gleich zu Beginn die Aufzählung möglicher Schreibarten zeigt, die u. a. sowohl Forkels Neuansatz einer an Affekten orientierten Stilklassifikation als auch die von Gottsched allgemein verbreitete und von Scheibe auf die Musik übertragenen Stilhöhenbegriffe „hoher", „mittlerer" und „niedriger" Stil ${ }^{59}$ enthält:

„Die musikalische Schreibart ist so verschieden wie die poetische. Sie kann erhaben und populär, einfältig und geschmückt, prächtig und simpel, hoch und niedrig, ernst und scherzend, tragisch und komisch, tiefsinnig und leicht $[\ldots]$ sein. ${ }^{60}$

Im weiteren Verlauf plädiert Schubart dann aber für eine stark reduzierte Zahl von Stilen. Die wünschenswerte Beschränkung auf die Unterscheidung von „religiösem“ und „profanem “ Stil sieht er allerdings, da „in neuern Zeiten die Musik in verschiedene Zweige“ sich „verbreitet hat" ${ }^{61}$ als nicht realisierbar an. So hält er schließlich an den drei „genera stylorum" fest - Kirchenstil, dramatischer Stil und Kammerstil, jeweils mit nur wenigen Subdivisionen -, ergänzt sie aber um den „Pantomimischen Stil“, also den Tanzstil.

Weniger innerhalb des Abschnitts zum Stil als vielmehr im Verlauf der Ausführungen zur Musikgeschichte lässt Schubart, offenbar Gedanken Marpurgs weiterführend, auch die Idee des Nationalstils in den Blick geraten. Während Mattheson die deutsche Musiklehre zugunsten des französischen und italienischen Stils herabwürdigt, formuliert Quantz prononciert, wenn „man die Musik der Deutschen, von mehr als einem Jahrhunderte her, genau untersuchet“, komme man zu dem Ergebnis, „daß sowohl ihr Geschmack als ihre Melodien, länger als bey ihren Nachbarn, ziemlich platt, trocken, mager und einfältig gewesen "62 seien, wohingegen es jetzt einen spezifischen deutschen Geschmack gebe, den er als neuen sog. ,vermischten Geschmack ${ }^{\star 63}$ propagiert. Demgegenüber proklamiert Marpurg „den Geschmack eines unsterblichen Leipziger Bachs“ als „einen besondern Originalgeschmack“, der nicht „aus der Nachahmung einer fremden Nation entstandenen"64 sei. Diese Vorstellung greift Schubart auf. Während in Heinses Hildegard von Hohenthal, 1795/96, also gut zehn Jahre nach der Niederschrift der Ideen erschienen, die damalig gegenwärtige deutsche Musik überhaupt keine Erwähnung findet, stellt Schubart, ebenfalls an Johann Sebastian Bach denkend, den er als „Orpheus der Deutschen "65 bezeichnet, die dezidierte These auf, Deutschland habe „Musiker hervorgebracht, die nicht bloß mit den Welschen wetteifern, sondern sie in Genie und Reichtum der Erfindung überflogen" "66 Schubart, der schon in seinem kleinen, zuerst in der Deutschen Chronik (1776) veröffentlichten Text Vom Nationalcharakter schreibt, „daß jeder Staat wie jeder einzelne Mensch seinen besonderen Charakter habe “" 67 geht aber insofern deutlich über Marpurg hinaus, als er die Wertschätzung der deutschen Musik in das Ineinssetzen von "musikalischem Geist" und „deutschem Charakter"68 überführt. Schubart hat damit einen Gedanken begründet, der in überhöhter Form noch in den

59 Johann Adolph Scheibe, Der Critische Musicus [Teil 1 (1737/1738), Hamburg 1738, Teil 2 (1739/1740), Hamburg 1740]. Neue vermehrte und verbesserte Auflage, Leipzig 1745, S. 125.

60 Schubart, Ideen, S. 348.

61 Ebd.

62 Johann Joachim Quantz, Versuch einer Anleitung, die Flöte traversière zu spielen. Faks.-Repr. der Ausgabe Berlin 1752, hrsg. von Horst Augsbach, Kassel u. a. 1992, S. $324 \mathrm{f}$.

63 Ebd., S. 332.

64 Friedrich Wilhem Marpurg, Der critische Musicus an der Spree, Bd. 1, Berlin 1750, S. 357.

65 Schubart, Ideen, S. 107.

66 Ebd., S. 45.

67 Chr. F. D. Schubart, Vom Nationalcharakter, in: ders., Vermischte Schriften (wie Anm. 24), S. 247.

68 Schubart, Ideen, S. 244. 
Schriften Thomas Manns begegnet, steht doch bei ihm, für den die Musik „die deutscheste der Künste “69 war, die Reflexion des Verhältnisses „von deutscher Musik, deutscher Identität und deutscher Geschichte“ im Brennpunkt seines „lebenslange[n] Nachdenken[s] über Musik“ ${ }^{70}$

\title{
4. Niedergang von Kirchenmusik und Orgelspiel
}

\subsection{Kirchenmusik}

Der Streit um die Frage, welche Musik dem Gottesdienst der christlichen Kirchen angemessen sei, ist gleichsam eine historische Konstante, gewinnt aber, was katholischerseits einmal mehr die Enzyklika Annus qui (1749) Benedikt XIV. dokumentiert, im 18. Jahrhundert aufgrund des Einflusses der Oper auch auf die kirchenmusikalischen Gattungen deutlich an Brisanz. Während u. a. Forkels der Kirchenmusik gewidmete Einleitung („Über Kirchenmusik und einige damit verwandte Gegenstände") zum zweiten Band seiner Musikgeschichte gleichermaßen wie E. T. A. Hoffmanns Aufsatz Alte und neue Kirchenmusik als zentrale Texte des ästhetischen Diskurses gelten, wird man den Namen Schubart in der entsprechenden Forschungsliteratur nur selten finden, was umso verwunderlicher ist, als die Kritik an der zeitgenössischen Kirchenmusikpflege sich bereits Jahre vor Forkel wie ein roter Faden durch Schubarts Schriften zieht, ${ }^{71}$ seine Situationsbeschreibungen - und hiermit antizipiert er Hoffmann - nicht, wie diejenigen Forkels oder Reichardts, konfessionsgebunden sind und es in der zweiten Hälfte des 18. Jahrhunderts, wie die nachfolgend zitierte Kritik an der Kirchenmusik am Pfälzischen Hof in Mannheim deutlich macht, kaum einen wortgewaltigeren Befürworter eines eigenständigen Kirchenstils gegeben haben dürfte.

\begin{abstract}
„Man würdigte dem Kirchenstyl nur weniger Aufmerksamkeit, verschmähte die alten Messen, und führte neue, im weichsten und winzigsten Opernstyle hingetändelte Kirchenmusik auf. Nichts ist profaner, als ein Lamm Gottes im girrenden neuwelschen Geschmack, ohne Himmelsgefühl hergelallt, und ein Kyrie, das in schnellen leichtfertigen Takten und Tönen, wie eine Theaternymphe daherfaselt. Ich trage den Verfall der Kirchenmusik so schwer auf dem Herzen, daß ich im Verfolge meiner Pilgerreise durch eine kleine Strecke Welt noch manches davon reden werde. "72
\end{abstract}

Für die Verweltlichung der Kirchenmusik sieht Schubart offenbar ganz bestimmte Personen ursächlich verantwortlich. Die Vereinigung der

„wetliche[n] Miene des Dramas mit dem Glutanlitze der Kirchenmusik [...] legte den ersten Grund zum Verfall der Musik. [...] An dieser Profanisierung ist niemand Schuld als die Großen, denn diese

69 Thomas Mann, Die Entstehung des Doktor Faustus. Roman eines Romans, in: Gesammelte Werke in 13 Bde., Bd. 11, Frankfurt a. Main 1974, S. 227.

70 Hans Rudolf Vaget, Seelenzauber. Thomas Mann und die Musik, Frankfurt a. M. 2006, S. 21.

71 Dass Schubart durch Martin Gerberts - allerdings ausgesprochen retrospektive, letztlich die Konzentration der liturgischen Musik auf den Gregorianischen Choral propagierende - Abhandlung De cantu et musica sacra a prima ecclesiae aetate usque ad praesens tempus, 2 Bde., St. Blasien 1774 (Faks.-Repr. Graz 1968, hrsg. und mit Registern versehen von Othmar Wessely [= Die großen Darstellungen der Musikgeschichte in Barock und Aufklärung, Bd. 4]) beeinflusst wurde, ist eher unwahrscheinlich, weil er laut Selbstaussage nur die musikhistorischen Werke Padre Martinis, Hawkins und Burneys rezipiert hat (s. o.). Zu Gerbert siehe Laurenz Lütteken, Die Rezeption päpstlicher Kirchenmusikverordnungen des 18. Jahrhunderts bei Martin Gerbert, in: Klaus Pietschmann (Hrsg.), Papsttum und Kirchenmusik vom Mittelalter bis zu Benedikt XVI. Positionen - Entwicklungen - Kontexte (= AnMl 47), Kassel usw. 2012, S. $190-200$.

72 Schubart, Schubart's Leben, S. 155. 
taten an die Genies die unsinnige Forderung, die Kirche aufs Theater und das Theater in die Kirche zu pflanzen." 73

Dass Schubart hier u. a. auf Mattheson abzielt, ist durchaus denkbar, plädierte der doch als prominentester Musikpublizist seiner Zeit für die Anwendung des dramatischen Stils auch in der Kirche. In diesem Zusammenhang ist vor allem Matthesons Reaktion Der neue Göttingische, aber viel schlechter, als die alten lacedämonischen, urtheilende Ephorus [...]“ (Hamburg 1727) auf die Streitschrift des Göttinger Gymnasialprofessors Joachim Meyer zu erwähnen, der unter dem Titel Unvorgreiffliche Gedancken über die Neulich eingerissene Theatralische Kirchen-Music Kritik an dem Neumeister-Typus der Kantate übte.

Der Hinweis auf „die alten Messen“ im obigen Zitat und die Begeisterung für die Psalmvertonungen Lassos, von denen es in der Aesthetik heißt, die Psalmen ließen sich kaum „besser in Musik übertragen “, ${ }^{74}$ lässt Schubarts Präferenz eines Stilideals erkennen, das in der Zeit vor der Stilwende um 1600 beheimatet ist, und zwar noch vor Heinse, der in Hildegard ebenfalls die Überlegenheit der sog. „alten Meister" thematisiert, ebenso wie vor Reichardt, der im von ihm in zwei Bänden herausgegebenen Musicalischen Kunstmagazin (1782 und 1791) sich umfangreicher mit der Kirchenmusik seiner Zeit auseinandersetzt, aber erst im zweiten Band die Musik der franko-flämischen Schule in den Blick nimmt. Schubarts Rekurs auf die sog. altklassische Kirchenmusik ist innerhalb des ästhetischen, außerkirchlich geführten Diskurses eine gänzlich neue Sicht und bedeutet einen substanziellen Unterschied zu Forkel, der den Verfall vorwiegend als institutionelles, in der kirchenmusikalischen Administration gründendes Problem sieht - Mangel an Sachkenntnis bei den Verantwortlichen in der Kirchenleitung und übertriebene Sparsamkeit ${ }^{75}$ - und eine auf einer historisierenden Perspektive basierende Problemlösungsstrategie vermeidet. Auch Forkel beklagt zwar, dass die „Unerfahrenheit der meisten Kirchenkomponisten, Musikdirektoren und Cantoren im wahren erbaulichen Kirchenstyl“ dazu führe, „daß sie kaum den Ausdruck jener Fröhlichkeit, die etwa in einem Tanzsaale herrscht, von derjenigen Gemüthstimmung, welche wir eine christliche Freudigkeit nennen, zu unterschieden wissen ". ${ }^{76}$ Forkels Lösungsweg ist jedoch nicht die Hinwendung zum Vergangenen, sondern, nach der Bewusstseinsbildung für die Bedeutung der Kirchenmusik, ${ }^{77}$ ganz praxisorientiert, der Appell an die Kirchenleitungen, sich um gutes Personal zu bemühen und entsprechende finanzielle Ressourcen zur Verfügung zu stellen. Eine expressis verbis formulierte Präferenz von mit einem bestimmten Stil verbundenen Satztechniken vermeidet er in gleicher Wiese wie einige Jahre vor ihm Quantz, der zwar der Überzeugung Ausdruck verliehen hat, Kirchenmusik erfordere „eine ernsthafte und andächtige Art der Composition, und der Ausführung“, diese jedoch nicht mit der Forderung nach alter Musik oder einer bestimmten Satztechnik verbindet.

Durchaus erwähnenswert ist, dass Forkel die Situation in der katholischen Kirche als deutlich besser schildert:

Schubart, Ideen, S. 50 .

Ebd.

75 Johann Nikolaus Forkel, Allgemeine Geschichte der Musik, Bd. 1, Leipzig 1788. Faks.-Repr., hrsg. von Claudia Maria Knispel, Laaber 2005, S. 26.

76 Ebd., S. 24

77 Siehe ebd., S. 48ff. 
„In den meisten Zeiten hat indessen die katholische Kirche für ihre Sänger und Spieler gesorgt. Man findet selten eine Stadtkirche in katholischen Ländern ohne besondere Stiftungen, die hauptsächlich, zur Besoldung der Kirchenmusiker bestimmt sind."78

Die Lektüre der Schriften Schubarts wirkt hier allerdings relativierend. Er besucht in München Messen, weil er „ein Ideal von Kirchenmusik im Herzen trug“, das er „hier gewiß realisiert zu finden glaubte " ${ }^{79}$ Enttäuscht muss er jedoch feststellen:

„Zwar hörte ich von Priestern und Chorknaben einige Antiphonen nach alter Manier - und trefflich vorgetragen; aber alle mit Instrumenten begleitete Musik war meistens profan. Der Sänger seufzte unter dem Sturme der Begleitung wie ein verirrtes Kind im Walde, drin der Sturmwind rast. Die Motiven waren meist der Oper entpflückt [...]. “80

Anders sieht Schubart aber offenbar die Situation des Orgelspiels in katholischen Kirchen. Im Erfassen und Darstellen des der jeweiligen liturgischen Situation entsprechenden Ausdrucks, so Schubart, seien „die Katholiken bei weitem, wenigstens der Zahl nach, unsere Meister, nachdem wir unser großes Muster, den unsterblichen Sebastian Bach, so weit aus den Augen verlieren, daß es kaum noch einen Menschen gibt, der seine Stücke spielen kann". 81

So sehr die Bevorzugung der Musik der sogenannten franko-flämischen Schule Schubart von Forkel unterscheidet, so sehr verweist sie auf Hoffmanns Idealisierung der „altitalienischen Vokalmusik“, von der Jürgen Heidrich meinte, dass hier „Ideen seines Lehrers Johann Friedrich Reichardt nachwirken “ ${ }^{82}$ Wenn Hoffmann mit dessen Werk auch bestens vertraut gewesen sein dürfte, kann kaum in Abrede gestellt werden, dass er ebenso die posthum publizierten Schriften Schubarts rezipiert hat und sein Blick auf die Kirchenmusik durch sie geprägt wurde, was die Beurteilung des kirchenmusikalischen Schaffens der Wiener Klassik vielleicht deutlich zu machen vermag. Zur Kirchenmusik Haydns schrieb Schubart:

„[...] nur tändelt er zuweilen, aus Vorneigung gegen den österreichischen Geschmack, auch in seinen Messen Verzierungen hin, die da nicht stehen sollten. Diese Flitter gleichen oft dem buntscheckigen Kleide des Harlekins, und entweihen das Pathos des Kirchenstyls. " 83

Ganz ähnlich formuliert Hoffmann:

„Mag es unverhohlen gesagt werden, daß selbst der, in seiner Art so große, unsterbliche J. Haydn, selbst der gewaltige Mozart, sich nicht rein erhielten von dieser ansteckenden Seuche des weltlichen, prunkenden Leichtsinns. Mozarts Messen [...] sind beinahe seine schwächsten Werke. [...] aber selbst in seinen ernstesten Werken für die Kirche hört sich Manches so, wie jene sich unter dem Tisch des Herrn beißenden Hunde erscheinen." 84

Wenn Schubart von Kirchenmusik spricht, sind zwei Begriffe von zentraler Bedeutung, und zwar „edle Einfalt“ und „Erhabenheit“. Der durch Winckelmann populär geworde-

78 Ebd., S. 26.

79 Schubart, Schubart's Leben, S. 190

80 Ebd.

81 Ebd., S. 99.

82 Jürgen Heidrich, Protestantische Kirchenmusikanschaung in der zweiten Hälfte des 18. Jahrbunderts. Studien zur Ideengeschichte ,wahrer Kirchenmusik', Göttingen 2001, S. 13.

83 Schubart, Ideen, S. 87f.

84 E. T. A. Hoffmann, Alte und neue Kirchenmusik [1814], in: ders., Fantasiestücke in Callot's Manier. Werke 1814, hrsg. von Hartmut Steinecke unter Mitarbeit von Gerhard Allroggen und Wulf Segebrecht (= ders., Sämtliche Werke, hrsg. von Hartmut Steinecke und Wulf Segebrecht unter Mitarbeit von Gerhard Allroggen u. a., Bd. 2/1), Frankfurt a. M. 1993, S. 523. 
ne, auf Verständlichkeit und Annehmlichkeit verweisende Begriff der „edlen Einfalt“ - er geht zurück auf den französischen Klassizismus und wurde schon 1739 von Mattheson auf die Musik übertragen ${ }^{85}$ - findet sich ebenso wie der der „Erhabenheit“ gleichermaßen bei Reichardt wie bei Hoffmann, in Schubarts Schriften aber ist er gleichsam eine Konstante und scheint - auch wenn er die „edle Einfalt" vor allem in den Psalmen Lassos und in Allegris Miserere realisiert sieht ${ }^{86}$ - eher auf ein künstlerisches Ideal zu verweisen als ein Epitheton klassischer Vokalpolyphonie zu sein, denn auch das aus Matthesons Regeln resultierende „Lied im Volkston“, wie es - Jahrzehnte später - von Schulz ${ }^{87}$ realisiert wurde, gehorcht bei Schubart der ästhetischen Prämisse der edlen Einfalt.

Statt des Begriffs „Erhabenheit“ kommt bei Schubart mitunter der der „Majestät“ ins Spiel. Den Psalmen Lassos z. B. spricht er "Einfalt, Hoheit, Majestät des Ausdrucks" ${ }^{\text {" } 88}$ zu. Tatsächlich scheint der Begriff des „Majestätischen“ treffender als der des „Erhabenen“ zu sein, denn verfolgt man die Begriffsgeschichte des „Erhabenen“, so kann, wie Edmund Burke gezeigt hat, das "Erhabene“ auch mit der Erfahrung des Bedrohlichen einhergehen. ${ }^{89}$ Hierfür sei im Hinblick auf die Musik beispielhaft Hoffmann genannt. In den Kreisleriana findet sich eine Notiz zu Bach, in der es heißt: „Musik! - mit geheimnisvollem Schauer, ja mit Grausen nenne ich dich! “90 Einige Jahre nach Schubart, und zwar in seinem 1788 publizierten Text Über die bildende Nachahmung des Schönen, bringt auch Karl Philipp Moritz die dem Erhabenen verwandte Kategorie des Majestätischen ins Spiel:

„Aus der höchsten Mischung des Schönen mit dem Edlen, da wo das äußere Schöne ganz im Ausdruck innerer Würde und Hoheit übergeht, erwächst der Begriff des Majestätischen. - Denken wir uns das Majestätische belebt, so muß es die Welt beherrschen, der Dinge Zusammenhang in sich fassen; der Erdkreis muß vor ihm sich beugen. ${ }^{91}$

Hier wird deutlich, dass dem Majestätischen weniger, wie Konrad Paul Liessmann meint, eine ,ethisch" als vielmehr eine nahezu religiös fundierte Hoheit zukommt. ${ }^{92}$ Als exemplum classicum dient Schubart das Miserere Allegris: „[...] so in die Einheit einer Himmelsempfindung verfößt, so in der vollen vierstimmigen Kraft und mit so auf der Herzenswage abgewogenen Tönen“, lässt es ihn darüber „Opern= und Kammerstyl, alle Schnörkel, Läufe, Vorschläge, Kadenzen [...] der neuesten Tonkuns “ ${ }^{\text {}}{ }^{3}$ vergessen.

\subsection{Orgelmusik}

Von Sachsen und Thüringen abgesehen, wo eine relativ große Schülerschaft Bachs tätig war, vollzog sich in der Orgelkomposition mindestens seit der Mitte des 18. Jahrhunderts

85 Der vollkommene Capellmeister, Hamburg 1739 (Studienausgabe Kassel usw. 1999), S. 143. Siehe hierzu Arno Forchert, Johann Sebastian Bach und seine Zeit, Laaber 2000, S. 251.

86 Schubart, Ideen, S. 50.

87 Johann Abraham Peter Schulz, Lieder im Volkston, bei dem Klaviere zu singen, 3 Teile, Berlin 1782-90.

88 Schubart, Ideen, S. 50.

89 Edmund Burke, Philosophische Untersuchungen über den Ursprung unserer Ideen vom Erhabenen und Schönen, übersetzt von Friedrich Bassenge, neu eingeleitet und hrsg. von Werner Strube, Hamburg 1980, S. 73.

90 E. T. A. Hoffmann, Fantasiestücke in Callot's Manier. III. Kreisleriana. 5. Höchst zerstreuet Gedanken, in: ders., Fantasiestücke in Callot's Manier. Werke 1814 [wie Anm. 84], S. 63.

91 Carl Philipp Moritz, Über die bildende Nachahmung des Schönen [1788], in: Werke, hrsg. von Horst Günther, Bd. 2, Frankfurt a. M. 1981, S. 549-578, hier: S. 559.

92 Konrad Paul Liessmann, Ästhetische Empfindungen, Wien 2009, S. 80.

93 Schubart, Schubart's Leben, S. 233. 
eine Abkehr von der polyphonen Gestaltung bei gleichzeitigem Eindringen von galanten Spielmanieren, wovon z. B. die "Orgelschule“ Knechts ${ }^{94}$ beredtes Zeugnis ablegt. Wo die Kontrapunktik als wesentliches Gestaltungsmittel erhalten blieb, tendierte sie, wie die Umschreibung Schubarts entsprechender Kompositionen Kirnbergers deutlich werden lässt, zu pretentiöser Kunstgelehrsamkeit: „Die Fugen von Kirnberger“ - nach Schubarts Auffassung „ein eiskalter Theoretiker“95 - „sind zwar schwerfällig und mühsam, aber doch mit großer Kunst gearbeitet [...]. ${ }^{96}$ Was diesen Werken in Schubarts Augen offenbar mangelt, ist eine mit der korrekten Handhabung eines bestimmten Regelwerks alleine nicht erreichbare ästhetische Qualität. So geht seine Kritik an der zeitgenössischen Kirchenmusik einher mit einer Klage über den Niedergang des Orgelspiels, der nicht zuletzt auch durch die Tatsache befördert wurde, dass in der zweiten Hälfte des 18. Jahrhunderts das ästhetische Gewicht sich endgültig von der Orgel auf die Saitenklaviere verlagerte. ${ }^{97}$ Schon während seines Nürnberg-Aufenthaltes 1756 stellt Schubart fest, nachdem er ,in den Kirchen [...] Schüler von dem deutschen Arion, dem unsterblichen Sebastian Bach"98 gehört hatte, „welch ein seltener Mann ein guter Orgelspieler sey". ${ }^{99}$ Und in den Ideen geißelt er das gängige Orgelspiel mit ähnlich harschen, bereits in der Deutschen Chronik von $1775 \mathrm{zu}$ findenden Worten, die auch sein Verdikt über die Kirchenmusik prägen:

„Es gibt keine Orgelspieler mehr! Da leiern sie das ganze Jahr ein ärmliches Präludium daher; spielen ihre Choräle ohne Empfindung; schlagen Dragonermärsche aus der Kirche, entweihen die Kommunion mit Vorspielen im Tone: Ach schläft denn alles schon, und Die Tochter soll ins Kloster gehen [...] Unsterblicher Geist des großen Sebastian Bachs, auf welchem Planeten bist Du?"100

In einem an Vogler gerichteten, 1786 auf dem Hohenasperg verfassten Schreiben formuliert Schubart eine kurze Ästhetik des Orgelspiels und des Organisten. Er rühmt „Hoheit und Würde“101 der Orgel, um anschließend zu beklagen, dass „dies gigantische Tongebäude, auf dessen Erfindung ein Engel stolzen könnte, in unsern betrübten Tagen von bleichgesichtigen Buben und jämmerlichen Stümpern so schrecklich mißhandelt wird“. ${ }^{102}$ Er beklagt „,[k]lavicembalische Hacker und Gaukler, Nachäffer des frechsten dramatischen Styls [...] freche Entweiher des hohen Kirchengesangs und unverständige Verächter des gebundenen Styls und des so mächtig wirkenden Contrapunkts [...] “. ${ }^{103}$ Wiewohl Schubart in Johann Adolph Scheibe einen „der gelehrtesten Musiker unseres Jahrhunderts" ${ }^{\text {"104 }}$ sah, ist kaum zu bezweifeln, dass er an die berühmte, die Fehde mit Birnbaum hervorrufende Satire dachte, die Scheibe auf die Musik Bachs verfasste. ${ }^{105}$ Anschließend entwirft Schubart, 15 Jahre vor Forkel ${ }^{106}$ und inhaltlich durchaus mit ihm vergleichbar, die Skizze des Ideals eines Orga-

94 Justin Heinrich Knecht, Vollständige Orgelschule, 3 Bde., Leipzig 1795-1798.

95 Schubart, Ideen, S. 92.

96 Ebd.

97 Arnfried Edler, Gattungen der Musik für Tasteninstrumente. Teil 2: Von 1750 bis 1830 (= Handbuch der musikalischen Gattungen, hrsg. von Siegfried Mauser, Bd. 7,2); Laaber 2003, S. 33.

98 Schubart, Schubart's Leben, S. 31.

99 Ebd.

100 Deutsche Chronik auf das Jahr 1774, hrsg. von Schubart [wie Anm. 12], S. 27.

101 Chr. F. D. Schubart, An Vogler, in: ders., Vermischte Schriften [wie Anm. 24], S. 61.

102 Ebd., S. 62.

103 Ebd.

104 Schubart, Ideen, S. 117.

105 In: J. A. Scheibe, Der Critische Musicus [wie Anm. 59], S. 55-65 und S. 833-858.

106 Siehe J. N. Forkel, Allgemeine Geschichte der Musik, Bd. 2, Leipzig 1801. Faks.-Repr., hrsg. von Claudia Maria Knispel, Laaber 2005, S. 42-46. 
nisten und kündigt schließlich an, in der „Aesthetik der Tonkunst [...] über das wahre Orgelspiel" sich „weitläufiger zu ergießen“. ${ }^{107}$ Der entsprechende Abschnitt bietet aber keine neuen Gesichtspunkte im Vergleich mit dem Brief an Vogler, wo es heißt:

„Der Orgelspieler muß [...] den Satz aus dem Grunde verstehen, muß die harmonischen Verhältnisse so ganz als möglich [...] jene so geheimnißvolle harmonische Ebb' und Fluth [...] völlig im Kopf und in der Faust haben; [...] Seine Phantasien müssen groß, neu, zweckgemäß sein[...]. Seine Vorspiele, Nachspiele und Zwischenspiele müssen dem Geiste der Orgel immer angemessen sein; müssen dem Hörer fromme Empfindungen wecken, erhalten, befestigen. Er muß daher alles von seinem Spiel absondern, was ganz ins Gebiet des Klavikords, bekielten Flügels, Fortepiano's [...] gehört." ${ }^{108}$

Erstaunen muss an diesem Text der Begriff des „Zweckmäßigen“, zeichnete sich doch schon zu Schubarts Zeit ab, dass die Zweckbestimmung ein Kriterium für ästhetische Belanglosigkeit war. Carl Philipp Moritz unterscheidet 1788 das „Schöne“ als das „in sich selbst Vollendete“ von dem nützlichen Gegenstand, der „bloß als Mittel“, 109 der Befriedigung zählt. Ihre definitive Formulierung findet die Idee des zweckfreien ästhetischen Wohlgefallens schließlich 1790 in Kants Kritik der Urteilskraft. Schubart versucht hier offenbar, das Junktim von Kunstwillen und Autonomie, wie es die großen, nicht mehr an Funktionen ausgerichteten, sondern quasi die Transzendierung der Gattung darstellende Choralbearbeitungen Bachs repräsentieren, aufzuheben und, um der kirchenmusikalischen Praxis willen, den Anspruch auf künstlerische Originalität und den der Funktionalität in Übereinstimmung zu bringen. Damit wendet er sich einem Problem zu, das im 19. Jahrhundert Franz Liszt in seinem Aufsatz Über zukünftige Kirchenmusik (1834) reflektieren sowie Jahrzehnte später mit seiner Komposition Via crucis (1878/79) beispielhaft zu lösen suchen wird.

\section{Bach-Rezeption}

Schubart gehört weder zu dem relativ engen Kreis, der Bach persönlich gekannt, noch zu denjenigen, die bei einem seiner Schüler studiert hat. Zudem lebte er weit entfernt von Berlin (Marpurg / Forkel) und Wien (von Swieten), den Zentren der zeitgenössischen BachRezeption. Und dennoch konnte er, wie der nachfolgend geschilderte Sachverhalt evident werden lässt, ein Bach-Bild zeichnen, welches sicherlich nicht ohne Einfluss auf das frühe 19. Jahrhundert gewesen ist.

Für Carl Dahlhaus war E. T. A. Hoffmann der „geschichtlich wirksamste, aber nicht der erste Ästhetiker, der Bachs Werk [...] für das Gedächtnis der Nachgeborenen zu retten versuchte". 110 Und er erwähnt einen 1801 in der Allgemeinen Musikalischen Zeitung erschienenen, von Triest, einem Prediger aus Stettin, verfassten Artikel, ${ }^{111}$ in dem Bach mit Newton verglichen wird. Den Vergleich mit Physiker Isaac Newton, der „zu einem bevorzugten Genie-Paradigma wurde" 112 übernahm Triest, so Dahlhaus, aus Hirschings Hand-

107 Schubart, An Vogler, (wie Anm. 101) S. 65.

108 Ebd., S. $63 f$.

109 Karl Philipp Moritz, Über den Begriff des in sich selbst Vollendeten [1785], in: Gesammelte Werke in 2 Bde, hrsg. von Jürgen Jahn, Berlin/München 1973, Bd. 2, S. 543.

110 Carl Dahlhaus, Zur Entstehung der romantischen Bach-Deutung [1978], in: ders., Klassische und romantische Musikästhetik, Laaber 1988, S. 127.

111 Johann Karl Friedrich Triest, Bemerkungen über die Tonkunst in Deutschland im achtzehnten Jahrhundert, in: $A M Z 3$ (1800/1801, Sp. 225ff.

112 Jochen Schmit, Die Geschichte des Genie-Gedankens in der deutschen Literatur, Philosophie und Politik, Bd. 1, Darmstadt 1998, S. 8. 
buch berübmter und denkwürdiger Personen, wo es heißt: „Was Newton als Weltweiser war, was Sebastian Bach als Tonkünstler. "113 Der ganze Abschnitt, in den dieser Satz eingebettet ist, zitiert allerdings, worauf bereits Schulze hingewiesen hat, ${ }^{114}$ wortwörtlich Schubart, und zwar die unter dem Stichwort „Sächsische Schule“ innerhalb des Geschichtsabschnitts der Ideen zu findenden Anmerkung zu Sebastian Bach - was Dahlhaus allerdings verborgen geblieben ist. Wiewohl die Ideen erst 1806 publiziert wurden, ist der Sachverhalt leicht zu erklären. Schubarts Sohn hatte, wie er in der Vorrede zur Ausgabe der Ideen schreibt, ${ }^{115}$ Ausschnitte bereits in verschiedenen Zeitschriften veröffentlicht, so auch in der Deutschen Monatsschrift. Aus eben dieser Zeitschrift konnte Hirsching zitieren, und zwar aus dem Jahrgang 1793, Stück 1, S. 85. Der Rückgriff Triests auf das Schubart-Zitat in Hirschings Handbuch legt schließlich auch die Annahme nahe, dass seine - allerdings schon bei Kirnberger aufscheinende - Charakterisierung Bachs als der „größte, tiefsinnigste Harmonist aller bisherigen Zeiten "116 ebenfalls weniger durch Reichardt, wie Dahlhaus annimmt, ${ }^{117}$ als vielmehr durch Schubart angeregt wurde, der die „kühne[n] Modulationen“ und die „so große Harmonie" als wesentliche Momente sieht, in denen sich das „Originalgenie eines Bachs" 118 manifestiere. Dahlhaus wertet diese Sätze Triests, die auch Hoffmann bekannt gewesen sein dürften, als „Bemerkungen, die nichts Geringeres als ein ästhetisch-geschichtsphilosophischer Traktat sind, in dem sich zum ersten Mal die Umrisse der Idee eines von Bach begründeten ,Zeitalters der deutschen Musik' abzeichnen “. ${ }^{119}$ Da die Triest'schen Ausführungen ganz offensichtlich ihren Ausgang nehmen von dem Bild, das Schubart von Bach gezeichnet hat, darf jener als Exponent einer Bach-Bewegung gelten, die, durchaus von Patriotismus getragen - Schubart bezeichnet Bach u. a. als „Orpheus der Deutschen" ${ }^{20}$-, in Forkels Bach-Biografie (1802) kulminiert. ${ }^{121}$ Nicht unerwähnt bleiben sollte in diesem Kontext aber auch, dass Schubarts Bach-Bild, insofern er die Kantaten propagiert, ein markantes Alleinstellungsmerkmal kennzeichnet. „Seine Jahrgänge, die er für die Kirche schrieb“, so Schubart, „trifft man jetzt äußerst selten an, ob sie gleich ein unerschöpflicher Schatz für den Musiker sind." ${ }^{122}$ Dieser Hinweis ist umso erstaunlicher, als im Bewusstsein der Nachwelt Bach bekanntlich zu allererst als Komponist von Instrumentalwerken existiert und in der zeitgenössischen Literatur zu Bach die Vokalmusik im Allgemeinen und die Kantaten im Besonderen, wohl nicht zuletzt der desolaten Quellensituation wegen, aber auch aufgrund der Distanz den vertonten Texten gegenüber, kaum Erwähnung finden.

113 Friedrich Karl Gottlob Hirsching, Historisch-literarisches Handbuch berühmter und denkwürdiger Personen, welche in dem 18. Jahrhunderte gestorben sind, 1. Abtlg. Bd. 1, Leipzig 1794, S. 79

114 Hans-Joachim Schulze, Dokumente zum Nachwirken Johann Sebastian Bachs 1750-1800 (= BachDokumente, Bd. III), Kassel/Basel/London 1984, S. 535, Dok. 987, Kom. II.

115 Schubart, Ideen, S. 6.

116 Zitiert nach: Carl Dahlhaus, Zur Entstehung der romantischen Bach-Deutung [wie Anm. 110], S. 128.

117 Siehe ebd.

118 Schubart, Ideen, S. 108.

119 Carl Dahlhaus, Zur Entstehung der romantischen Bach-Deutung, S. 127.

120 Schubart, Ideen, S. 107.

121 Siehe Carl Dahlhaus, Zur Entstehung der romantischen Bach-Deutung, S. 125.

122 Schubart, Ideen, S. 108. 


\section{Schluss}

Die eigentliche Zielsetzung seiner Ästhetik skizziert Schubart am Ende seiner Einleitung:

„Vor allen Dingen [...] muß der musikalische Aesthetiker den Wirkungen der Tonkunst sorgfältig nachspüren, und nach richtigen Principien zu zeigen wissen, warum dieser oder jene Gang so große und einschneidende Wirkung hervorbringe, und warum ein anderer Satz kraftlos vom Herzen der Menschen abgleite." 123

Eine ästhetische Urteile fundierende, für eine Beantwortung der in der „Einleitung“ projizierten leitenden Fragen „[W]as ist das musikalische Schöne? wie wird dieß Schöne hervorgebracht?" 124 unverzichtbare Kriteriologie bleibt Schubart schuldig, was einmal mehr von der Schwierigkeit zeugt, den Rang einer Komposition in der satztechnischen Faktur nachweisen zu wollen. Dass die Beschreibung des Schaffens einzelner Komponisten im Ungefähren verbleibt, ist die Kehrseite dieses Defizits.

Trotz eines solchen durchaus markanten Mankos, trotz der rezeptionshistorisch zwar relativ erfolgreichen, aber hinterfragbaren Tonartencharakteristik und trotz all ihrer Vorläufig keit sind Schubarts Ideen, wie ich meine, bisher nicht adäquat gewürdigt worden, beinhalten sie doch Aspekte, die, sofern man sie überhaupt zur Kenntnis nahm, in ihrer Bedeutung für die Zukunft des ästhetisch-historischen Denkens unterschätzt wurden und deshalb eine grundlegende Neubewertung der allgemein auf die Rolle des Stürmers und Drängers reduzierten Person Schubarts geboten scheinen lassen.

Die zentralen Aspekte möchte ich abschließend aus dem bisher Gesagten thesenartig extrahieren:

1. Eine Ästhetik der Musik, wie sie mit Schuberts Ideen erstmals publiziert wurde, ist Symptom eines neuen musikalischen Selbstbewusstseins, das durchaus konvergiert mit dem in den beiden letzten Dekaden des 18. Jahrhunderts vor allem in den Schriften Carl Philipp Moritz' greifbar werdenden Autonomiegedankens der Kunst.

2. Bei Schubart zeigen sich erstmals in der Musikhistoriografie das bis dahin gültige teleologische Fortschrittsmodell substituierende Ansätze zu einem ästhetisch-historischen Relativismus, dem zufolge prinzipiell jede Kultur ihre eigene, gleichberechtigte Idee von Schönheit besitzt.

3. Schubart revolutioniert das Ausdrucksprinzip, indem er die Darstellung der persönlichen Befindlichkeit des musizierenden Subjekts an die Stelle bloßer Mimikri treten lässt, wie sie letztlich von C. P. E. Bach propagiert wurde.

4. Während die im 18. Jahrhundert weit verbreitete Diskussion des „welschen “ und „französischen" Stils auf disponiblen Kunststilen beruhte, verweist Schubart bereits, und zwar unter Anerkennung des Anderen und Fremden und damit ohne Hypostasierung, auf die im 19. Jahrhundert vorherrschende Idee eines Nationalstils, der die Vorstellung zugrundelag, in der Musik könne sich der Geist eines Volkes niederschlagen.

5. In einer Zeit, in der die Orgelmusik zur Bedeutungslosigkeit herabzusinken und die Orgel zum bloßen Andachtsgenerator zu degenerieren drohte, erinnerte Schubart nicht nur an den Vorbildcharakter ihrer technischen Beherrschung durch J. S. Bach und seinen 
dem Instrument am ehesten adäquaten kontrapunktischen Stil, sondern verwies darüber hinaus mit Verve auch auf die ontologische Dignität der Orgel.

6. Während E. T. A. Hoffmann in erheblichem Umfang ältere Denkweisen reflektiert, kommt Schubart das Verdienst zu, abgesehen von der äußerst lebendigen Beschreibung der zeitgenössischen kirchenmusikalischen Realität, den Keim für die Ideengeschichte einer auf der sogenannten "altklassischen Vokalpolyphonie" basierenden "wahren“ Kirchenmusik gepflanzt zu haben, ohne dabei jedoch der Gefahr einer ideologischen Verengung zu erliegen, wie sie später vor allem in den kirchenmusikalischen Reformbewegungen greifbar wird.

7. Mit der für seine Zeit eher untypischen gleichzeitigen Wertschätzung für die Musik der franko-flämischen Schule und J. S. Bachs ${ }^{125}$ antizipiert Schubart E. T. A. Hoffmann, der in seinen Musikalischen Novellen schreibt:

„Man stritt heute viel über unsern Sebastian Bach und über die alten Italiener, man konnte sich durchaus nicht vereinigen, wem der Vorzug gebühre. Da sagte mein geistreicher Freund: „Sebastian Bachs Musik verhält sich zu der Musik der alten Italiener wie das Münster zu Straßburg zu der Peterskirche in Rom. Wie tief hat mich das wahre, lebendige Bild ergriffen."126

8. An der Schwelle zum 19. Jahrhundert dürften die - uneingeschränkte Bewunderung ausdrückenden - Gedanken Schubarts zu J. S. Bach, nicht zuletzt auch im Hinblick auf E. T. A. Hoffmann, von zentraler publizistischer Wirkung gewesen sein, wurden sie doch mehrfach zitiert und in unterschiedlichen Zeitschriften veröffentlicht.

125 Vgl. z. B. Martin Gerbert (siehe Anm. 71).

126 E. T. A. Hoffmann, Fantasiestücke in Callot's Manier. III. Kreisleriana. 5. Höchst zerstreuet Gedanken, in: ders., Fantasiestücke in Callot's Manier. Werke 1814 [wie Anm. 84], S. 62. 